\title{
Conservative management of tracheal rupture in a child after blunt trauma
}

\author{
Gonul Kucuk, M.D. ${ }^{a}$, Ufuk Ates, M.D. ${ }^{a}$, Gulnur Gollu, M.D. ${ }^{a}$ and Aydin Yagmurlu, M.D. ${ }^{a}$
}

\begin{abstract}
Tracheal rupture following blunt trauma is rare but is potentially life threatening. Usually surgical treatment is suggested for tracheal rupture however recently conservative management has also been reported for patients with stable vital signs and stable ventilatory state. The aim of this study is to report a case with tracheal rupture following a blunt trauma to the neck after a bicycle accident that was managed conservatively.

Key words: tracheal disease, neck injuries, wounds, nonpenetrating.
\end{abstract}

http:/ /dx.doi.org/10.5546/aap.2016.eng.e454

\section{INTRODUCTION}

Tracheal rupture is a rare condition but potentially life threatening. The incidence of traumatic airway injury is $0.4 \%$ for blunt traumas. ${ }^{1}$ They usually occur after blunt trauma but can also occur after severe coughing, vomiting or may also be iatrogenic secondary to stenosis dilatation, rigid bronchoscopy or tracheal intubation. ${ }^{2}$ The diagnosis is usually delayed or missed. However recently reports on tracheal rupture have increased in number because of improved care of trauma patients. ${ }^{3}$ Stable patients with the diagnosis of tracheal rupture might undergo unnecessary surgical treatment, which also increases mortality and morbidity. The objective is to present a six-year old boy with trachea rupture who was managed conservatively with favorable outcome.

\section{CASE REPORT}

A six-year old boy who had bicycle accident with direct trauma from the handlebars was admitted to hospital with pain and swelling in the neck. The physical examination revealed

a. Ankara University School of Medicine. Department of Pediatric Surgery, Ankara, Turkey.

E-mail Address:

Gulnur Gollu, M.D.: drggollu@yahoo.com

Funding: None.

Conflict of interest: None.

Received: 5-9-2016

Accepted: 6-23-2016 subcutaneous emphysema spreading from the mandibula down to sternum, ribs and scapula. Hemodynamics and ventilatory parameters including oximetry were normal. Chest x-ray revealed subcutaneous emphysema and pneumomediastinum (Figure 1). Thoracic computed tomography (CT) (Figure 2) and virtual bronchoscopy (Figure 3) revealed subcutaneous emphysema, pneumomediastinum and longitudinal tracheal rupture of $1.5 \mathrm{~cm}$ in length, which was $2 \mathrm{~cm}$ above the carina at the posterior wall of the trachea. Under close monitorization of clinical and ventilatory parameters a cannula was inserted on the sternum to drain subcutaneous emphysema. He was supported with nasal oxygen during the first day. The patient received $100 \mathrm{mg} / \mathrm{kg} /$ day intravenous cephalosporin for antibiotic therapy and intravenous paracetamol for analgesia. The cannula was left for four days to drain subcutaneous emphysema and his control chest X-ray was normal on fourth day. He received intravenous antibiotics for six days. The patient recovered rapidly and he was clinically stable and chest X-ray was normal; he was discharged on sixth day. The patient had no problem in his follow-ups of three years. He was clinically stable, didn't have any respiratory distress or stridor and the chest $\mathrm{X}$-rays were normal during the follow-ups. Informed consent was obtained from the parents of the child for publication.

FIgure 1: Chest $x$-ray. Subcutaneous emphysema and pneumomediastinum

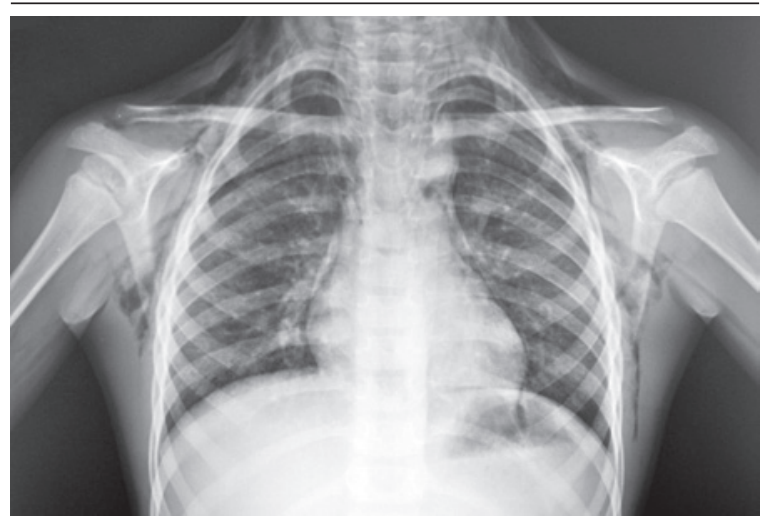




\section{DISCUSSION}

Tracheal ruptures are rare injuries with high mortality and morbidity. ${ }^{3}$ Trachea is usually damaged due to one of three mechanisms: high energy trauma to the chest while the glottis is closed, acceleration- or deceleration-type injury or blow against the neck during flexion or hyperextension. ${ }^{3,4}$ Minor trauma may also lead to tracheal rupture especially in the membranous part of the trachea, the weakest part of laryngotracheal complex. The rupture may be transverse, longitudinal or complex. ${ }^{3}$

Subcutaneous emphysema is the most specific sign of tracheal disruption. ${ }^{4}$ It should be suspected in the presence of pneumomediastinum with or without pneumothorax, hemoptysis and subcutaneous emphysema. ${ }^{3}$ Subcutaneous emphysema is the evidence of a lesion of the airway but is not always evident in initial examination. The patients with stable ventilator state should undergo radiological evaluation. Chest X-ray might show subcutaneous emphysema, pneumothorax or pneumomediastinum. ${ }^{4}$ If there is a suspicion of tracheal rupture and the patient is in stable condition, thoracic CT and virtual bronchoscopy, which is a three-dimensional image reconstructed by the scans of multislice helical CT, should be performed. This technique is less invasive than tracheoscopy which has a risk of worsening the condition especially at the time of anesthetics induction during manual ventilation by increasing the airway pressure. ${ }^{4}$ Conservative treatment includes antibiotics and analgesics administration, oxygen and emphysema drainage with a cannula or catheter. Respiratory distress is the main determining factor in the management. Extensive wounds with important air leakage with unstable condition are managed by surgical repair.

Nakamori et al., also reported a case of tracheal rupture diagnosed using virtual bronchoscopy. ${ }^{5}$ Kaloud et al. recommended surgical repair in ruptures with a length exceeding $1 \mathrm{~cm} .{ }^{6}$ Kuhne et al., Merol et al. and D'Odement et al. reported successful conservative treatment of tracheal lesions. ${ }^{3,4,7}$ Most of the authors perform bronchoscopy for the diagnosis of rupture and these authors do not perform a CT scan since they consider it as unnecessary procedure. However bronchoscopy, which is a possibly risky examination, might be unnecessary in well-tolerated lesions. Merol et al. also considers that tracheoscopy should not be in the initial management in stable patients with obvious diagnosis depending on clinical examination, X-ray films and CT scan. ${ }^{4}$

Finally, conservative management should be held in mind in cases with minor trauma, stable condition and accurate diagnosis. The most important and most difficult part of tracheal rupture is the accurate diagnosis of injury. Follow-up is very important in patients with tracheal rupture since there is a risk of tracheal stenosis. Wood et al. published the largest case series for the outcomes of conservative treatment of non-penetrating traumatic tracheal injuries in children and they stated that five of eight patients who had tracheal rupture developed stenosis in two-year follow-up and three of these patients required further surgery. ${ }^{8}$ Although most authors recommend surgical treatment in tracheobronchial lesions, conservative
FIGURE 2. Thoracic computed tomography. Tracheal rupture (White arrow)

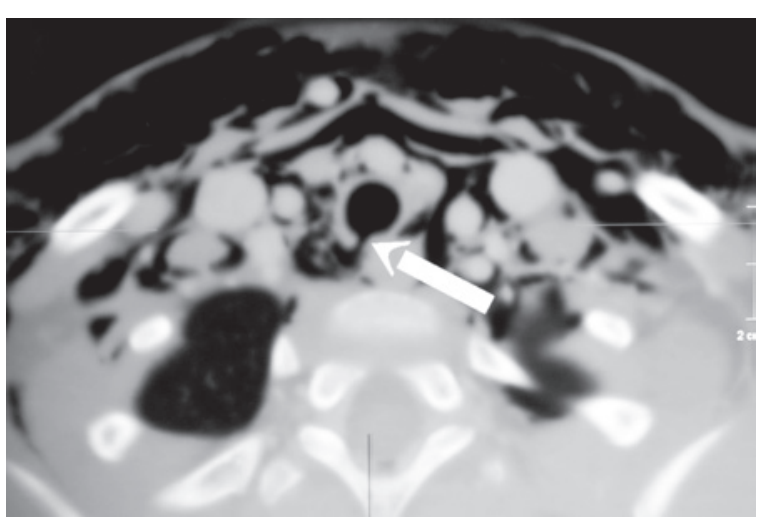

FIGURE 3. Virtual bronchoscopy. Longitudinal tracheal rupture $2 \mathrm{~cm}$ above the carina at posterior wall of the trachea

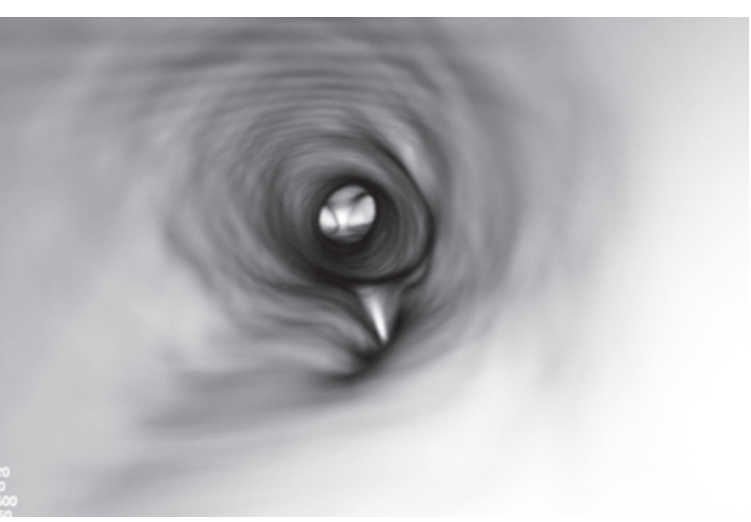


management is also practicable especially in well tolerated lesions by close monitorization of ventilatory and hemodynamic parameters. Virtual bronchoscopy should be performed in good condition patients in order to avoid unnecessary risky examination for those who might be treated without any invasive procedure and avoid open surgical procedures in most of stable patients.

\section{REFERENCES}

1. Prokakis C, Koletsis EN, Dedeilias P, Fligou F, et al. Airway trauma: a review on epidemology, mechanisms of injury, diagnosis and treatment. J Cardiothoracac Surg 2014;9:117.

2. StevensMS,MullisTC,CarronJD.Spontaneoustracheal rupture caused by vomiting. Am J Otolaryngol 2010;31(4):276-8.
3. Kuhne CA, Kaiser GM, Flohe S, Beiderlinden M, et al. Nonoperative management of tracheobronchial injuries in severely injured patients. Surg Today 2005;35(7):518-23.

4. Poli-Merol ML, Belouadah M, Parvy F, Chauvet P, et al. Tracheobronchial injury by blunt trauma in children: Is emergency tracheobronchoscopy always necessary? Eur J Pediatr Surg 2003;13(6):398-402.

5. Nakamori Y, Hayakata T, Fujimi S, Satou K, et al. Tracheal rupture diagnosed with virtual bronchoscopy and managed nonoperatively: a case report. J Trauma 2002;53(2):369-71.

6. Kaloud H,Smolle-JuettnerFM,Prause G, ListWF. Iatrogenic ruptures of tracheobronchial tree. Chest 1997;112(3):774-8.

7. D'Odemont JP, Pringot J, Goncette L, Goenen M, et al. Spontaneous favorable outcome of tracheal laceration. Chest 1991;99(5):1290-2.

8. Wood JW, Thornton B, Brown CS, Mclevy JD, et al. Traumatic tracheal injury in children: a case series supporting conservative management. Int J Pediatr Otorhinolaryngol 2015;79(5):716-20. 